\title{
Approximate aggregation of linear discrete models with two time scales: re-scaling slow processes to the fast scale
}

\author{
Tri Nguyen-Huu ${ }^{\mathrm{ab} *}$, Rafael Bravo de la Parra ${ }^{\mathrm{ac}}$ and Pierre Auger ${ }^{\mathrm{ab}}$ \\ ${ }^{a}$ UMMISCO IRD, 32, Av. Henri Varagnat, 93143 Bondy Cedex, France; ${ }^{b}$ IXXI, 7 rue du Vercors, \\ 69007 Lyon, France; ${ }^{c}$ Departamento de Matemáticas, Universidad de Alcalá, 28871 \\ Alcalá de Henares (Madrid), Spain
}

(Received 11 June 2009; final version received 1 October 2009)

\begin{abstract}
Mathematical models used in ecology often inherit the complexity found in nature and thus are governed by a large number of variables. Aggregation of variables methods is used to make such models mathematically tractable by building an approximate system governing fewer variables. We extend here aggregation methods for linear discrete models with processes occurring at different time scales. In practical cases, some processes that occur at a fast time scale are often only measured at a slow time scale, like mortality. We present a general class of models with two time scales involving such kind of processes. We show how they should be re-scaled in order to be taken into account at the fast time scale in a more realistic approach. The approximate aggregation of these models is undertaken and justified in mathematical terms. We also provide an application to a model of a structured population in a two-patch environment.
\end{abstract}

Keywords: aggregation of variables; time scales; population dynamics; linear discrete models

\section{Introduction}

The representation of complex ecological systems by means of mathematical models makes the latter to be also complex and thus requiring some reduction to be mathematically tractable. This complexity arises from the fact that observations and model predictions are all related to a certain scale and a detailed model necessarily includes several different scales. To avoid incompatibility of ecological data and to translate model processes from one to another scale, we need transferring information between scales, what is called scaling. Hierarchy theory provides the conceptual framework of how the involved processes and components interrelate and how they can be ordered $[10,16]$. It deals with a ranking of levels, each consisting of interacting entities with their own dynamics, being the strength of the interactions among the entities, expressed by the size of process rates, what is most helpful for scaling issues. The entities of a given level with strong or fast interactions can be grouped giving rise to the entities at next level. In this context, the process of up-scaling consists in deriving aggregated variables and their dynamics from the lower level.

From a mathematical point of view, aggregation of variables was introduced in the field of economics and was made prevalent in ecology in Ref. [8]. The concept was further extended to approximated aggregation in Ref. [9]. Approximate aggregation techniques

*Corresponding author. Email: tri.nguyen-huu@ird.fr 
have been widely studied in the context of models with different time scales, see the reviews in Refs. [1,2]. In this work, we are interested in linear discrete models. In Refs. $[3,13-15]$, linear discrete models with two time scales are studied. The models include two processes acting at different time scales and the analysis makes profit of the fast process (strong interactions) to find the global (aggregated) variables and build up the reduced system governing approximately their dynamics. It is proved that the elements defining the asymptotic behaviour of the complete and the aggregated systems are equal up to a certain order. In Ref. [3], the time unit of the complete system is the one associated with the fast dynamics, while in Ref. [13], it is the one corresponding to the slow process.

In the construction of a discrete model with two time scales, it is not always possible to choose as time unit the fast one because it may happen that during a fast time unit, the action of the slow process is not describable while, on the other hand, if the system is expressed in the slow time unit, the fast process can be represented by letting it act a number $k$ of times reflecting the ratio between time scales. If we represent the slow process by matrix $S$ and the fast process by matrix $F$, then the matrix of the complete system is $S F^{k}$. This generic form has been used to model the dynamics of an age or stage-structured population inhabiting a patchy environment, e.g. multi-regional Leslie models (trouts in a river network in Ref. [5], insects in Brittany landscape in Ref. [12]) where matrix $F$ corresponds to the migration process and gathers dispersal matrices for every age class, and matrix $S$ that places together the patch-specific survival and migration rates of a classical Leslie matrix for each patch. It is further assumed that migration is fast in comparison to local demographic processes (reproduction, mortality and ageing) which can happen once a year while individuals could migrate from one to another patch every week. By supposing that $k$ tends to infinity, i.e. the fast dynamics is instantaneous with respect to the slow one, the model reduces to a classical Leslie model with the equilibria of the migration process reflected in its parameters. In Ref. [13], it is proved that the eigen-elements defining the asymptotic behaviour of the complete system can be approximated from the corresponding elements of the aggregated system.

A drawback of the described slow-fast multi-regional Leslie model is that it may be unrealistic in some ecological situations. It is assumed that individuals at first perform a series of $k$ dispersal events, and then reproduction and survival occur according to the arrival patch, that is, individuals move frequently from patch to patch but nothing happens concerning reproduction or mortality meanwhile. Though the assumption is realistic for reproduction, because discrete models are mainly used for species having offspring once every time unit, it is not so in the case of mortality, since individuals may die at any time with different survival rates playing a role. It is more realistic to take into account survival together with migration at the fast time scale and reproduction and ageing at the slow time scale. Let $L$ and $M$ be the matrices representing the demographic and migration processes, respectively. The matrix associated to the described slow-fast multi-regional Leslie model is $L M^{k}$. If we write $L=R S$ where $R$ is a matrix representing ageing and reproduction and $S$ is survival, the more realistic model that we propose has the following associated matrix: $R\left(S^{1 / k} M\right)^{k}$. Because a survival matrix is usually a diagonal matrix, we have denoted $S^{1 / k}$ the diagonal matrix whose diagonal elements are the fractional powers of the diagonal elements of $S$ for a better understanding. We have re-scaled survival to fit at the fast scale in the form $S^{1 / k}$.

The aim of this work is to extend the results in Ref. [13] to a larger class of models encompassing situations where it is needed to re-scale a part of the slow process at the fast scale. In general, the aggregation of a system consists in defining a small number of global variables, functions of all state variables and to derive a system describing their dynamics. 
A linear discrete system can be perfectly aggregated whenever the associated matrix of order $N$ can be written as the product of two matrices of dimensions $N \times q$ and $q \times N$, respectively, with $q<N$. If this is the case, a new system with $q$ variables can be defined and the dynamics of both systems can be obtained from each other. We are interested in approximate aggregation and so, from an abstract point of view, we are treating linear discrete systems which are closed to systems that admit perfect aggregation. Section 2 includes the presentation of a general reducible linear discrete system, defined by means of a sequence of matrices whose limit when $k$ tends to infinity admits perfect aggregation, together with the construction of its aggregated system and the relationship between their corresponding asymptotic elements. In Section 3, we apply the general framework of Section 2 to a linear discrete population model whose dynamics is driven by two processes, slow and fast, whose corresponding characteristic time scales are very different from each other. It is also presented as a particular case a model that includes the situations where we have to re-scale a part of the slow process at the fast scale. Section 4 illustrates this latter case applying the aggregation method to a simple model of a population structured into juvenile and adult classes and migrating between two patches. After the conclusions, there is an appendix where a nice result is proved on the convergence of a matrix sequence which shows that the general method applies to the models presented at the end of Section 3 and in Section 4.

\section{Reducible linear discrete population models}

In this section, we present a general discrete population model, let us call it complete model, which will be susceptible to being reduced.

The model evolves in discrete time and is defined by a convergent sequence of non-negative matrices $H_{k} \in \mathbb{R}_{+}^{N \times N}$, with $k \in \mathbb{N}$. Denoting by $X_{k, n}=\left(x_{k, n}^{1}, \ldots, x_{k, n}^{N}\right) \in \mathbb{R}^{N}$ the vector of state variables at time $n$, the complete system is defined by

$$
X_{k, n+1}=H_{k} X_{k, n}
$$

In order to reduce the system (1), we will suppose that $k$ is large enough and we impose some conditions which are specified in the two following hypotheses:

Hypothesis 2.1. There exists a matrix $\bar{H}$ which is the limit of $H_{k}$

$$
\lim _{k \rightarrow \infty} H_{k}=\bar{H} \text {. }
$$

In the second hypothesis, we use the concept of allowability of a non-negative matrix in Ref. [17]. A non-negative matrix is called row allowable (column allowable) if it has, at least, one positive entry in each row (column).

Hypothesis 2.2. There exist $q \in \mathbb{N}, q<N$, and two non-negative matrices $G \in \mathbb{R}_{+}^{q \times N}$ column allowable and $D \in \mathbb{R}_{+}^{N \times q}$ row allowable such that $\bar{H}$ can be expressed as

$$
\bar{H}=D G .
$$

In what follows, we suppose that Hypotheses 2.1 and 2.2 are met. Hypothesis 2.2 is usually met when dealing with iterations of primitive stochastic matrices. In that case, the Perron-Frobenius theorem is useful to determine the decomposition of matrix $\bar{H}$. 
Then, we proceed to reduce system (1) in two steps. First, we define the so-called auxiliary system which approximates (1) when $k \rightarrow \infty$. Denoting its vector of variables at time $n$ by $X_{n}$, this auxiliary system reads

$$
X_{n+1}=\bar{H} X_{n}=D G X_{n} .
$$

Secondly, we define the so-called global variables, which will play the role of state variables of the reduced, or aggregated, system

$$
Y_{n}:=G X_{n} \in \mathbb{R}^{q} .
$$

Multiplying both sides of (2) by $G$, we obtain

$$
Y_{n+1}=G X_{n+1}=G D G X_{n}=G D Y_{n},
$$

which is the linear discrete system for the global variables $Y_{n}$ that we use as approximation of system (1). Defining $A=G D$, the aggregated system reads

$$
Y_{n+1}=A Y_{n} \text {. }
$$

Notice that, through the previous procedure, we have constructed an approximation of (1) that allows us to reduce a system with $N$ variables to a new system with $q$ variables. In most practical applications, $q$ will be much smaller than $N$.

\subsection{Relationships between the complete and the aggregated system}

This section is devoted to the study of the relationships between the aggregated (3) and the original (1) systems. We will start relating the spectral properties of matrices $\bar{H}$ and $A$, associated to auxiliary (2) and aggregated (3) systems, and then we will compare the elements governing the asymptotic behaviours of complete (1) and auxiliary (2) systems by considering matrix $H_{k}$ as a perturbation of matrix $\bar{H}$.

Let us notice first that the relationship between the auxiliary and the aggregated systems is an example of perfect aggregation in the sense of Ref. [8]. To be precise, if $\left\{X_{n}\right\}_{n \in \mathbb{N}}$ is the solution of auxiliary system (2) associated to the initial condition $X_{0}$ and $\left\{Y_{n}\right\}_{n \in \mathbb{N}}$ the solution of aggregated system (3) for $Y_{0}=G X_{0}$, then they verify $X_{n}=D Y_{n-1}$ and $Y_{n}=G X_{n}$.

To compare the asymptotic behaviour of the auxiliary and the aggregated systems, we assume that matrix $A$ of the aggregated system is primitive, what implies, theorem of Perron-Frobenius [17], that there exists $\lambda_{A}>0$ which is its strictly dominant eigenvalue, with corresponding positive right column eigenvector $\mathbf{v}_{A}$ and positive left row eigenvector $\mathbf{u}_{A}$. These eigen-elements characterize the asymptotic behaviour of the aggregated system (3) in the following sense: for every initial state, we have that, in the long term, the population size grows exponentially at a rate $\lambda_{A}$, the population reaches a stable structure given by the vector $\mathbf{v}_{A}$, and the reproductive values [4] associated to the different population classes or stages are given by vector $\mathbf{u}_{A}$.

We can now establish in the following theorem the relationship of the eigen-elements characterizing the asymptotic behaviour in the auxiliary and the aggregated systems.

\section{Proposition 2.3. If matrix $A$ is primitive then}

(1) Matrix $\bar{H}$ is also primitive and $\lambda_{A}$ is its strictly dominant eigenvalue.

(2) Vector $\mathbf{v}_{\bar{H}}=D \mathbf{v}_{A}$ is a positive right column eigenvector of $\bar{H}$ associated to $\lambda_{A}$.

(3) Vector $\mathbf{u}_{\bar{H}}=\mathbf{u}_{A} G$ is a positive left row eigenvector of $\bar{H}$ associated to $\lambda_{A}$. 
Proof.

(1) $A$ primitive implies (Ref. [7], Theorem 8.5.2) that there exists a $p \in \mathbb{N}$ such that $A^{p}>0$. We have now that $(\bar{H})^{p+1}=D A^{p} G$ is positive due to Hypothesis 2.2 and so $\bar{H}$ is primitive.

Once we prove that $\lambda_{A}$ is an eigenvalue of $\bar{H}$ with a positive associated eigenvector, Corollary 8.1.30 in Ref. [7] implies that $\lambda_{A}=\rho(\bar{H})$ and thus its strictly dominant eigenvalue.

(2) Matrix $D$ being row allowable (Hypothesis 2.2) ensures that $\mathbf{v}_{\bar{H}}=D \mathbf{v}_{A}$ is positive and to show that it is an eigenvector associated to $\lambda_{A}$ :

$$
\bar{H} \mathbf{v}_{\bar{H}}=D G D \mathbf{v}_{A}=D A \mathbf{v}_{A}=D \lambda_{A} \mathbf{v}_{A}=\lambda_{A} \mathbf{v}_{\bar{H}}
$$

(3) Matrix $G$ being column allowable (Hypothesis 2.2) ensures that $\mathbf{u}_{\bar{H}}=\mathbf{u}_{A} G>0$ and we also have

$$
\mathbf{u}_{\bar{H}} \bar{H}=\mathbf{u}_{A} G D G=\mathbf{u}_{A} A G=\lambda_{A} \mathbf{u}_{A} G=\lambda_{A} \mathbf{u}_{\bar{H}},
$$

i.e. $\mathbf{u}_{\bar{H}}$ is a left row eigenvector of $\bar{H}$ associated to $\lambda_{A}$.

Matrix $H_{k}$ of the complete system can be considered as a perturbation of matrix $\bar{H}$ of the auxiliary system

$$
H_{k}=\bar{H}+\left(H_{k}-\bar{H}\right)
$$

where matrix $H_{k}-\bar{H}$ converges to zero as $k$ tends to infinity (Hypothesis 2.1). Therefore, the higher the value of $k$, the closer are the eigenvalues and eigenvectors of $H_{k}$ and $\bar{H}$. Thus, if $k$ is large enough we know about the asymptotic behaviour of the complete system from that of the auxiliary one and, through Proposition 2.3, from that of the aggregated one.

THeOREM 2.4. Suppose that Hypotheses 2.1 and 2.2 are verified, and that matrix $A$ of the aggregated system (3) is primitive, $\lambda_{A}$ being its strictly dominant eigenvalue, and $\mathbf{v}_{A}$ and $\mathbf{u}_{A}$ its corresponding positive right column and left row eigenvectors, respectively. Then, we can conclude that, for $k$ large enough, matrix $H_{k}$ of the complete system (1) is primitive and, for $k \rightarrow \infty$ and any consistent norm $\|\cdot\|$, its strictly dominant eigenvalue $\lambda_{H_{k}}$, and its corresponding positive right column and left row eigenvectors $\mathbf{v}_{H_{k}}$ and $\mathbf{u}_{H_{k}}$ verify:

(1) $\lambda_{H_{k}}=\lambda_{A}+\frac{\mathbf{u}_{A} G\left(H_{k}-\bar{H}\right) D \mathbf{v}_{A}}{\lambda_{A} \mathbf{u}_{A} \mathbf{v}_{A}}+O\left(\left\|H_{k}-\bar{H}\right\|^{2}\right)$.

(2) $\mathbf{v}_{H_{k}}=D \mathbf{v}_{A}+O\left(\left\|H_{k}-\bar{H}\right\|\right)$.

(3) $\mathbf{u}_{H_{k}}=\mathbf{u}_{A} G+O\left(\left\|H_{k}-\bar{H}\right\|\right)$.

Proof. Proposition 2.3 establishes the primitivity of matrix $\bar{H}$ that together with Hypothesis 2.1 implies that, for $k$ large enough, matrix $H_{k}$ has, at least, the same positive entries that $\bar{H}$ and so that it is also primitive (Ref. [7], Theorem 8.5.3).

The rest of the proof is a direct consequence of Proposition 2.3, considering $H_{k}$ a perturbation of $\bar{H},(4)$, and using the results on matrix perturbations in Ref. [18] (pp. 183 and 240). 
We have shown that we can get essential information about the asymptotic behaviour of the complete system (growth rate, stable distribution and reproductive values) through the study of the aggregated system.

\section{Linear discrete population models with two time scales}

In this section, we apply the general framework of Section 2 to a linear discrete population model whose dynamics is driven by two processes, slow and fast, whose corresponding characteristic time scales are very different from each other. The population considered is divided into $q$ groups, and each group $i(i=1, \ldots, q)$ divided into $N^{i}$ subgroups, with $N=N^{1}+\cdots+N^{q}$. This population structure, as we will see below, is directly related to the two different processes and their different associated time scales. We represent the state of the population at time $n$ by vector $X_{n}:=\left(\mathbf{x}_{n}^{1}, \ldots, \mathbf{x}_{n}^{q}\right)^{\mathrm{T}} \in \mathbb{R}_{+}^{N}$, where $\mathrm{T}$ denotes transposition. Every vector $\mathbf{x}_{n}^{i}:=\left(x_{n}^{i 1}, \ldots, x_{n}^{i N^{i}}\right) \in \mathbb{R}_{+}^{N^{i}}$ represents the state of the $i$ group.

The slow process is defined by the non-negative projection matrix $S \in \mathbb{R}_{+}^{N \times N}$, which we consider divided into blocks $S^{i j}, 1 \leq i, j \leq q$. We have then

$$
S=\left[\begin{array}{cccc}
S^{11} & S^{12} & \cdots & S^{1 q} \\
S^{21} & S^{22} & \cdots & S^{2 q} \\
\vdots & \vdots & \ddots & \vdots \\
S^{q 1} & S^{q 2} & \cdots & S^{q q}
\end{array}\right],
$$

where each block $S^{i j}=\left[S_{i j}^{m l}\right]$ has dimensions $N_{i} \times N_{j}$ and characterizes the rates of transference of individuals from the subgroups of group $j$ to those of group $i$. More specifically, for each $m=1,2, \ldots, N_{i}$ and each $l=1,2, \ldots, N_{j}, S_{i j}^{m l}$ represents the rate of transference of individuals, due to the slow process, from subgroup $l$ of group $j$ to subgroup $m$ of group $i$ throughout a slow time unit.

We assume fast process to be internal for each group $i(i=1, \ldots, q)$. Individuals interact strongly among the subgroups of the same group while these interactions are weaker with individuals in other groups. For each group $i$, the fast process is defined by a sequence of non-negative matrices $F_{i, k} \in \mathbb{R}_{+}^{N_{i} \times N_{i}}$ with $k \in \mathbb{N}$, where $k$ represents the ratio of the slow to the fast time scale and $F_{i, k}$ the projection matrix through a fast time unit for the specific $k$ and the group $i$. The fast process for the whole population is then defined by the sequence of block-diagonal matrices $F_{k}=\operatorname{diag}\left\{F_{1, k}, \ldots, F_{q, k}\right\} \in \mathbb{R}_{+}^{N \times N}$.

We choose as time step of the complete model that corresponding to the slow dynamics and in order to approximate the effect of the fast process over a time interval much larger than its own, we assume that during this time step the fast process acts $k$ times before the slow process acts, where $k$ in applications will take a large value. Therefore, the complete system is defined by

$$
X_{k, n+1}=S\left(F_{k}\right)^{k} X_{k, n},
$$

where $\left(F_{k}\right)^{k}$ denotes the $k$-power of matrix $F_{k}$.

In order to meet Hypotheses 2.1 and 2.2, we make the following assumptions.

Hypothesis 3.1. Fast process: for every $i(i=1, \ldots, q)$, there exists a matrix $\bar{F}_{i}=$ $\lim _{k \rightarrow \infty}\left(F_{i, k}\right)^{k}$ and two positive matrices $G_{i} \in \mathbb{R}_{+}^{1 \times N_{i}}$ and $E_{i} \in \mathbb{R}_{+}^{N_{i} \times 1}$ such that $\bar{F}_{i}=E_{i} G_{i}$. Slow process: for every $i(i=1, \ldots, q)$, matrix $S_{i i}$ is row allowable.

We denote $\bar{F}=\operatorname{diag}\left\{\bar{F}_{1}, \ldots, \bar{F}_{q}\right\} \in \mathbb{R}_{+}^{N \times N}, G=\operatorname{diag}\left\{G_{1}, \ldots, G_{q}\right\} \in \mathbb{R}_{+}^{q \times N}$ and $E=$ $\operatorname{diag}\left\{E_{1}, \ldots, E_{q}\right\} \in \mathbb{R}_{+}^{N \times q}$. Then, we have $\lim _{k \rightarrow \infty}\left(F_{k}\right)^{k}=\bar{F}=E G$. 
Assuming Hypothesis 3.1, we follow the development of Section 2 in the particular case of system (5) where $H_{k}=S\left(F_{k}\right)^{k}, \bar{H}=S \bar{F}$ and $D=S E$. The auxiliary system is $X_{n+1}=S \bar{F} X_{n}=S E G X_{n}$ and we define the global variables

$$
Y_{n}=\left(Y_{1, n}, \ldots, Y_{q, n}\right):=\left(G_{1} \mathbf{x}_{n}^{1}, \ldots, G_{q} \mathbf{x}_{n}^{q}\right)=G X_{n} .
$$

The fast process, apart from being internal for each group, attains an equilibrium which for an initial distribution among its subgroups $\mathbf{x}_{0}^{i}$ is $\bar{F}_{i} \mathbf{x}_{0}^{i}$ where we can notice that this equilibrium, $\bar{F}_{i} \mathbf{x}_{0}^{i}=E_{i} G_{i} \mathbf{x}_{0}^{i}$, depends on initial conditions only through the value of the associated global variable $G_{i} \mathbf{x}_{0}^{i}$.

The aggregated system, denoting $\bar{S}=G S E$, reads

$$
Y_{n+1}=\bar{S} Y_{n} .
$$

Finally, as a direct consequence of Theorem 2.4, we obtain the following result that allows us to study the asymptotic behaviour of system (5) by means of the aggregated system (7).

Proposition 3.2. Suppose that Hypothesis 3.1 is verified, and that matrix $\bar{S}$ of the aggregated system (7) is primitive, its strictly dominant eigenvalue being $\lambda_{\bar{S}}$, and $\mathbf{v}_{\bar{S}}$ and $\mathbf{u}_{\bar{S}}$ its corresponding positive right column and left row eigenvectors, respectively. Then, we can conclude that, for $k$ large enough, matrix $S\left(F_{k}\right)^{k}$ of the complete system (5) is primitive and, for $k \rightarrow \infty$ and any consistent norm $\|\cdot\|$, its strictly dominant eigenvalue $\lambda_{S\left(F_{k}\right)^{k}}$, and its corresponding positive right column and left row eigenvectors $\mathbf{v}_{S\left(F_{k}\right)^{k}}$ and $\mathbf{u}_{S\left(F_{k}\right)^{k}}$ verify

(1) $\lambda_{S\left(F_{k}\right)^{k}}=\lambda_{\bar{S}}+\frac{\mathbf{u}_{\bar{S}} G S\left(\left(F_{k}\right)^{k}-\bar{F}\right) S E \mathbf{v}_{\bar{S}}}{\lambda_{\bar{S}} \mathbf{u}_{\bar{S}} \overline{\mathbf{v}}_{\bar{S}}}+O\left(\left\|\left(F_{k}\right)^{k}-\bar{F}\right\|^{2}\right)$.

(2) $\mathbf{v}_{S\left(F_{k}\right)^{k}}=S E \mathbf{v}_{\bar{S}}+O\left(\left\|\left(F_{k}\right)^{k}-\bar{F}\right\|\right)$.

(3) $\mathbf{u}_{S\left(F_{k}\right)^{k}}=\mathbf{u}_{\bar{S}} G+O\left(\left\|\left(F_{k}\right)^{k}-\bar{F}\right\|\right)$.

We propose in the sequel, a particular case of fast dynamics for which Hypothesis 3.1 is met. We suppose, for every $i=1, \ldots, q$, that matrix $F_{i, k}$ is the product of two matrices:

$$
F_{i, k}=D_{i, k} M_{i}
$$

Matrices $D_{i, k}$ are defined from a non-singular matrix $D_{i}$ of order $N^{i}$ as $D_{i, k}=\exp \left((1 / k) D_{i}^{\prime}\right)$, where $D_{i}^{\prime}$ is a matrix such that $\exp \left(D_{i}^{\prime}\right)=D_{i}$. Matrix $M_{i}$ is a primitive matrix of order $N^{i}$ whose strictly dominant eigenvalue is 1 with a positive left-associated eigenvector, row vector $\mathbf{u}_{i}=\left(u_{i, 1}, \ldots, u_{i, N^{i}}\right)$ and a positive rightassociated eigenvector, column vector $\mathbf{v}_{i}=\left(v_{i, 1}, \ldots, v_{i, N^{i}}\right)^{\mathrm{T}}$ verifying $\mathbf{u}_{i} \cdot \mathbf{v}_{i}=1$. Now, Theorem A.1 (see Appendix) applies and we have

$$
\lim _{k \rightarrow \infty}\left(D_{i, k} M_{i}\right)^{k}=\gamma_{i} \mathbf{v}_{i} \mathbf{u}_{i} \quad \text { with } \gamma_{i}=\exp \left(\mathbf{u}_{\mathbf{i}} D_{i}^{\prime} \mathbf{v}_{\mathbf{i}}\right)
$$

and

$$
\left\|\left(D_{i, k} M_{i}\right)^{k}-\gamma_{i} \mathbf{v}_{i} \mathbf{u}_{i}\right\|=O\left(\frac{1}{k}\right) .
$$

Therefore, calling $\bar{F}_{i}=\lim _{k \rightarrow \infty}\left(D_{i, k} M_{i}\right)^{k}, G_{i}=\mathbf{u}_{i}$ and $E_{i}=\gamma_{i} \mathbf{v}_{i}$, we see that Hypothesis 3.1 is met and that we can estimate the convergence in Proposition (3.2) in terms of $1 / k$

$$
\left\|\left(F_{k}\right)^{k}-\bar{F}\right\|=O\left(\frac{1}{k}\right) .
$$


We present in Section 4 an application of this case to an age-structured population inhabiting a multi-patch environment.

\section{Application}

The model we present in this section includes, on one hand, the most basic life cycle division [11], the population is structured into two classes: non-reproducing juveniles and reproducing adults, and, on the other hand, the simplest heterogeneous environment, which is composed of just two different patches. The demography is defined by the survival rates of juveniles and adults, the maturation rates of juveniles and the fertility rates of adults.

For each patch $j(j=1,2)$, let $\sigma_{1 j}$ and $\sigma_{2 j}$ be the fraction of the juveniles and adults (respectively) alive at time $n$ that survive to time $n+1$. Also, let $\mu_{j}$ be the fraction of the surviving juveniles that mature to become adults. Finally, supposing that reproduction happens at the end of each period of time $[n, n+1)$, let $\phi_{j}$ be the number of juveniles produced by an adult that survive to time $n+1$. The projection matrix corresponding to patch $j$ thus reads as follows:

$$
\left(\begin{array}{cc}
\sigma_{1 j}\left(1-\mu_{j}\right) & \sigma_{2 j} \phi_{j} \\
\sigma_{1 j} \mu_{j} & \sigma_{2 j}
\end{array}\right) .
$$

The complete model includes demography together with migrations between patches. Migrations are considered fast in comparison to demography, and the time unit of the associated discrete system is the one associated to demography. We consider reproduction and maturation as processes that happen once at the end of each period of time $[n, n+1)$ but, on the other hand, mortality could happen at any time between $n$ and $n+1$. In order to take this into account and to combine demography and migrations, which occur $k$ times in each period of time, we write matrix (10) in the following form:

$$
\left(\begin{array}{cc}
\sigma_{1 j}\left(1-\mu_{j}\right) & \sigma_{2 j} \phi_{j} \\
\sigma_{1 j} \mu_{j} & \sigma_{2 j}
\end{array}\right)=\left(\begin{array}{cc}
1-\mu_{j} & \phi_{j} \\
\mu_{j} & 1
\end{array}\right)\left(\begin{array}{cc}
\left(\sigma_{1 j}\right)^{\frac{1}{k}} & 0 \\
0 & \left(\sigma_{2 j}\right)^{\frac{1}{k}}
\end{array}\right)^{k} .
$$

We represent the state of the population at time $n$ by vector

$$
X_{n}:=\left(x_{n}^{11}, x_{n}^{12}, x_{n}^{21}, x_{n}^{22}\right)^{\mathrm{T}} \in \mathbb{R}_{+}^{4},
$$

where $x^{1 j}$ and $x^{2 j}$ denote respectively the density of juveniles and adults in patch $j$.

The projection matrix of migrations for an interval of time which is $1 / k$ of the one associated to demography, representing $k$ the ratio between time scales, follows:

$$
\left(\begin{array}{cccc}
1-q_{1} & p_{1} & 0 & 0 \\
q_{1} & 1-p_{1} & 0 & 0 \\
0 & 0 & 1-q_{2} & p_{2} \\
0 & 0 & q_{2} & 1-p_{2}
\end{array}\right)
$$

where $q_{1}$ and $p_{1}$ represent the proportions of juveniles which leave respectively patches 1 and 2 , and $q_{2}$ and $p_{2}$ the proportions of adults which leave, respectively, patches 1 and 2 . 
Now putting together demography and migrations and having in mind the order of the variables in $X_{n}$ and the decomposition in (11), we propose the following complete model:

$$
X_{n+1}=S\left(F_{k}\right)^{k} X_{n}
$$

where

$$
S=\left(\begin{array}{cccc}
1-\mu_{1} & 0 & \phi_{1} & 0 \\
0 & 1-\mu_{2} & 0 & \phi_{2} \\
\mu_{1} & 0 & 1 & 0 \\
0 & \mu_{2} & 0 & 1
\end{array}\right)
$$

and

$$
F_{k}=\left(\begin{array}{cccc}
\left(\sigma_{11}\right)^{\frac{1}{k}} & 0 & 0 & 0 \\
0 & \left(\sigma_{12}\right)^{\frac{1}{k}} & 0 & 0 \\
0 & 0 & \left(\sigma_{21}\right)^{\frac{1}{k}} & 0 \\
0 & 0 & 0 & \left(\sigma_{22}\right)^{\frac{1}{k}}
\end{array}\right)\left(\begin{array}{cccc}
1-q_{1} & p_{1} & 0 & 0 \\
q_{1} & 1-p_{1} & 0 & 0 \\
0 & 0 & 1-q_{2} & p_{2} \\
0 & 0 & q_{2} & 1-p_{2}
\end{array}\right)
$$

Following the notation in (8), we have that

$$
D_{i, k}=\left(\begin{array}{cc}
\left(\sigma_{i 1}\right)^{\frac{1}{k}} & 0 \\
0 & \left(\sigma_{i 2}\right)^{\frac{1}{k}}
\end{array}\right) \quad \text { and } \quad M_{i}=\left(\begin{array}{cc}
1-q_{i} & p_{i} \\
q_{i} & 1-p_{i}
\end{array}\right)
$$

We note then that $D_{i}=\operatorname{diag}\left\{\sigma_{i 1}, \sigma_{i 2}\right\}$ and so $D_{i}^{\prime}=\operatorname{diag}\left\{\ln \left(\sigma_{i 1}\right), \ln \left(\sigma_{i 2}\right)\right\}$. Matrices $M_{i}$, provided that $p_{i}, q_{i} \in(0,1)$, are stochastic and primitive, and we can choose the eigenvectors associated to eigenvalue 1 to be

$$
\mathbf{u}_{i}=(1,1) \quad \text { and } \quad \mathbf{v}_{i}=\left(\frac{p_{i}}{p_{i}+q_{i}}, \frac{q_{i}}{p_{i}+q_{i}}\right)^{\mathrm{T}} .
$$

We can now calculate

$$
\gamma_{i}=\exp \left(\mathbf{u}_{i} D_{i}^{\prime} \mathbf{v}_{i}\right)=\left(\sigma_{i 1}\right)^{\frac{p_{i}}{p_{i}+q_{i}}}\left(\sigma_{i 2}\right)^{\frac{q_{i}}{p_{i}+q_{i}}}
$$

matrix $G=\operatorname{diag}\left\{\mathbf{u}_{1}, \mathbf{u}_{2}\right\}$ and matrix $E=\operatorname{diag}\left\{\gamma_{1} \mathbf{v}_{1}, \gamma_{2} \mathbf{v}_{2}\right\}$. The global variables

$$
Y_{n}=\left(Y_{1, n}, Y_{2, n}\right)=G X_{n}=\left(x_{n}^{11}+x_{n}^{12}, x_{n}^{21}+x_{n}^{22}\right)^{\mathrm{T}} \in \mathbb{R}_{+}^{2}
$$

are the total densities of juveniles and adults, respectively, and the resulting aggregated 
system is

$$
Y_{n+1}=\operatorname{GSEY}_{n}=\left(\begin{array}{cc}
\gamma_{1} \frac{p_{1}\left(1-\mu_{1}\right)+q_{1}\left(1-\mu_{2}\right)}{p_{1}+q_{1}} & \gamma_{2} \frac{p_{2} \phi_{1}+q_{2} \phi_{2}}{p_{2}+q_{2}} \\
\gamma_{1} \frac{p_{1} \mu_{1}+q_{1} \mu_{2}}{p_{1}+q_{1}} & \gamma_{2}
\end{array}\right) Y_{n}
$$

Proposition 3.2 tells us that the dominant eigen-elements of system (13) can be approximated from the corresponding eigen-elements of system (14) with a discrepancy which is, according to expression (9), a $O(1 / k)$. To illustrate the kind of results that one can deduce, we introduce a simplifying assumption in system (13). We suppose that we are treating the case of a precocious and iteroparous species, e.g. small mammals and birds, see Ref. [11], which begin reproducing when a year old and survive and reproduce for several years. To introduce this assumption into the model, we set $\mu_{1}=\mu_{2}=1$, all surviving juveniles become adults in a period of time. The projection matrix of the aggregated system (14) is now

$$
\left(\begin{array}{cc}
0 & \gamma_{2} \frac{p_{2} \phi_{1}+q_{2} \phi_{2}}{p_{2}+q_{2}} \\
\gamma_{1} & \gamma_{2}
\end{array}\right)
$$

If we calculate $n$, the inherent net reproductive number of this matrix, see Ref. [6], which shares with its strictly dominant eigenvalue being larger, equal or less than 1

$$
n=\frac{\gamma_{1} \gamma_{2}}{1-\gamma_{2}} \cdot \frac{p_{2} \phi_{1}+q_{2} \phi_{2}}{p_{2}+q_{2}}
$$

We obtain (Ref. [6], Theorem 1.1.3) that solutions of the aggregated system grow exponentially if $n>1$ and decay exponentially if $n>1$. Via Proposition 3.2 we get, for $k$ large enough, the same conclusions on the solutions of complete system (13) in the case $\mu_{1}=\mu_{2}=1$. Though we presented a model with two patches, no added difficulty would have been met in a similar model with a large number of patches. The aggregated system together with the conditions on exponential growth or extinction of the population could have been expressed as simply as in the two patches case.

\section{Discussion}

In the present work, we have introduced a very general class of linear discrete models that admit an approximate reduction. We have extended previous works $[3,13,14]$ on aggregation methods by showing how the aggregated model is built and how the asymptotic behaviour of the original model can be studied through it.

When there are two processes acting at different time scales that should be gathered in a single discrete model, there is a decision to make on the time unit to use. If we use the fast time scale, then we face the problem of describing the action of the slow process along a time unit much smaller than its own. This problem is, in many situations, unsolvable because there are many discrete processes that have no sense if observed at time intervals different from the ones they are naturally associated to. On the other hand, the choice of the slow time scale avoids this problem because we just need to let the fast process act 
a number of times approximating the ratio between time scales in order to describe its action through a slow time unit.

Discrete models in population dynamics involving two time scales use to classify processes as those which occur at slow time scale and those which occur at fast time scale. Nevertheless, some processes such as mortality, predation and others, which are often measured at slow time scale, act almost continuously and thus should be better considered as occurring at fast time scale. For this kind of processes, we have shown how to express their actions on a much shorter interval of time by performing a sort of $k$ th root of their known actions, whenever this has sense as in the proposed application in Section 4 with mortality. Once the originally slow process has been re-scaled to the fast time scale, the conditions on fast dynamics that allow the reduction of the model change with respect to those appearing in previous works. In Section 2, we have proposed these conditions in their most abstract setting Hypotheses 2.1 and 2.2. We have shown how to build the aggregated model and determined its asymptotic relationship with the original model. Section 3 particularizes the general setting of Section 2 so that models with two time scales, which appear in previous works, are included and the option of re-scaling some of the considered slow processes is also admitted.

We have illustrated the results in Section 3 with an example in Section 4 that emphasizes the fact that mortality is measured at slow time scale but represented in the model at fast time scale. Even a simple application like the one proposed shows that aggregation methods provide analytic results that could not be obtained otherwise just by working with the complete model.

Further methodological studies should consider cases where Hypothesis 2.1 is generalized. An important case where Hypothesis 2.1 fails to be met is the case where migration matrices are not primitive, leading to cycles at fast time scale. These kinds of matrices appear in seasonal migrations. A real world application is planned for a spatially explicit population dynamics model of the species Abax parallelepipedus in Brittany landscape, for which the spatial dimension has only been considered implicitly in Ref. [12].

\section{Acknowledgements}

R. Bravo de la Parra and P. Auger were partially supported by Ministerio de Educación y Ciencia (Spain), project MTM2008-06462-C02-01, and FEDER.

\section{References}

[1] P. Auger, R. Bravo de la Parra, J.C. Poggiale, E. Sánchez, and T. Nguyen-Huu, Aggregation of Variables and Applications to Population Dynamics, Lecture Notes in Mathematics 1936, Mathematical Biosciences Subseries, Springer Verlag, Berlin, 2008.

[2] P. Auger, R. Bravo de la Parra, J.C. Poggiale, E. Sánchez, and L. Sanz, Aggregation methods in dynamical systems and applications in population and community dynamics, Phys. Life Rev. 5 (2008), pp. 79-105.

[3] R. Bravo de la Parra, P. Auger, and E. Sánchez, Aggregation methods in discrete models, J. Biol. Syst. 3 (1995), pp. 603-612.

[4] H. Caswell, Matrix Population Models, Sinauer Associates, Inc., Sunderland, 2001.

[5] S. Charles, R. Bravo de la Parra, J.P. Mallet, H. Persat, and P. Auger, Population dynamics modelling in an hierarchical arborescent river network: An attempt with Salmo trutta, Acta Biotheor. 46 (1998), pp. 223-234.

[6] J.M. Cushing, An Introduction to Structured Population Dynamics, SIAM, Philadelphia, 1998.

[7] R.A. Horn and C.A. Johnson, Matrix Analysis, Cambridge University Press, Cambridge, 1985.

[8] Y. Iwasa, V. Andreasen, and S. Levin, Aggregation in model ecosystems I: Perfect aggregation, Ecol. Model. 37 (1987), pp. 287-302. 
[9] Y. Iwasa, S. Levin, and V. Andreasen, Aggregation in model ecosystems II: Approximate aggregation, Math. Med. Biol. 6 (1989), pp. 1-23.

[10] H. Lischke, T.J. Löffler, P.E. Thornton, and N.E. Zimmermann, Model up-scaling in landscape research, in A Changing World. Challenges for Landscape Research, F. Kienast, O. Wildi, and S. Ghosh, eds., Vol. 8, Springer Landscape Series, Dordrecht, 2007, pp. 249-272.

[11] M.G. Neubert and H. Caswell, Density-dependent vital rates and their population dynamic consequences, J. Math. Biol. 41 (2000), pp. 103-121.

[12] J.B. Pichancourt, F. Burel, and P. Auger, Assessing the effect of habitat fragmentation on population dynamics: An implicit modeling approach, Ecol. Model. 192 (2006), pp. 543-556.

[13] E. Sánchez, R. Bravo de la Parra, and P. Auger, Discrete models with different time-scales, Acta Biotheor. 43 (1995), pp. 465-479.

[14] L. Sanz and R. Bravo de la Parra, Variables aggregation in a time discrete linear model, Math. Biosci. 157 (1999), pp. 111-146.

[15] L. Sanz and R. Bravo de la Parra, Approximate reduction techniques in population models with two time scales: Study of the approximation, Acta Biotheor. 50 (2002), pp. 297-322.

[16] D.C. Schneider, The rise of the concept of scale in ecology, Bioscience 51(7) (2001), pp. 545-553.

[17] E. Seneta, Non-Negative Matrices and Markov Chains, Springer Verlag, New York, 1981.

[18] G.W. Stewart and J.I. Guang Sun, Matrix Perturbation Theory, Boston Academic Press, Boston, 1990.

\section{Appendix A. The convergence of a matrix sequence}

- Let $M$ be a non-negative matrix of order $n$, primitive and whose strictly dominant eigenvalue is 1 .

The Perron-Frobenius theorem yields that eigenvalue 1 is simple, has a positive left-associated eigenvector, row vector $\mathbf{u}=\left(u_{1}, \ldots, u_{n}\right), \mathbf{u} M=\mathbf{u}$, and a positive right-associated eigenvector, column vector $\mathbf{v}=\left(v_{1}, \ldots, v_{n}\right)^{\mathrm{T}}, M \mathbf{v}=\mathbf{v}$, which can be chosen to verify $\mathbf{u} \cdot \mathbf{v}=1$. In this case, the limit of the powers of matrix $M$ exists, we call it $\bar{M}$, and can be expressed in terms of $\mathbf{u}$ and $\mathbf{v}, \bar{M}=\lim _{k \rightarrow \infty} M^{k}=\mathbf{v u}$. Matrix $\bar{M}$ represents the eigenprojection associated to 1 , so

$$
M \bar{M}=\bar{M} M=\bar{M} \bar{M}=\bar{M} .
$$

and also matrix $M-\bar{M}$ has the same eigenvalues than $M$ except that 1 is substituted by 0 , therefore its spectral radius verifies $\rho(M-\bar{M})<1$ and thus there exists a norm $\|\cdot\|$ in $\mathbb{R}^{n}$ such that for the associated matrix norm in $\mathbb{R}^{n \times n}, \mid\|\cdot\| \|$, holds

$$
|| M-\bar{M}|| \mid<r<1 \quad \text { and }|||M|||=1 .
$$

- Let $D$ be a non-singular matrix of order $n$ and $D^{\prime}$ a matrix such that $\exp \left(D^{\prime}\right)=D$, then let us define matrices $D_{k}=\exp \left((1 / k) D^{\prime}\right)$.

Matrix $D_{k}$ can be written as

$$
D_{k}=I+P_{k} \quad \text { with }\left|\left\|P_{k} \mid\right\|=O\left(\frac{1}{k}\right)\right.
$$

and as

$$
D_{k}=I+\frac{1}{k} D^{\prime}+N_{k} \quad \text { with }\left|\left\|N_{k} \mid\right\|=O\left(\frac{1}{k^{2}}\right)\right.
$$


Matrix $\delta D$, for $\delta>0$, verifies $\delta D=\exp \left(D^{\prime}+(\log \delta) I\right)$, and so we denote

$$
(\delta D)^{\prime}=D^{\prime}+(\log \delta) I \quad \text { and } \quad(\delta D)_{k}=\exp \left(\frac{1}{k}\left(D^{\prime}+(\log \delta) I\right)\right) .
$$

We then have for any $k=0,1,2, \ldots$ :

$$
(\delta D)_{k}=\exp \left(\frac{1}{k} D^{\prime}\right) \exp \left(\left(\frac{1}{k} \log \delta\right) I\right)=\delta^{\frac{1}{k}} D_{k}
$$

TheOREM A.1. Let $M$ and $D$ be matrices as described previously. Then,

$$
\lim _{k \rightarrow \infty}\left(D_{k} M\right)^{k}=\lim _{k \rightarrow \infty}\left(M D_{k}\right)^{k}=\gamma \bar{M},
$$

with $\gamma=\exp \left(\mathbf{u} D^{\prime} \mathbf{v}\right), \quad$ and, furthermore, $\left\|\left(M D_{k}\right)^{k}-\gamma \bar{M}\right\|=O(1 / k)$ and $\|\left(D_{k} M\right)^{k}-$ $\gamma \bar{M} \|=O(1 / k)$.

Proof. The facts that $\left(D_{k} M\right)^{k}=D_{k}\left(M D_{k}\right)^{k}\left(D_{k}\right)^{-1}$ and $\lim _{k \rightarrow \infty} D_{k}=\lim _{k \rightarrow \infty}\left(D_{k}\right)^{-1}=I$ yield that $\lim _{k \rightarrow \infty}\left(D_{k} M\right)^{k}=\lim _{k \rightarrow \infty}\left(M D_{k}\right)^{k}$, so we just need to prove, by the equivalence of norms, that

$$
\|\|\left(M D_{k}\right)^{k}-\gamma \bar{M} \mid \|=O\left(\frac{1}{k}\right)
$$

which is equivalent to $\left\|\left(D_{k} M\right)^{k}-\gamma \bar{M}\right\|=O(1 / k)$.

If we prove (A6) for matrix $\delta D$, for any $\delta>0$, we will have it also proved for $D$ because, on the one hand,

$$
\begin{aligned}
\left(M(\delta D)_{k}\right)^{k} & =\left(M \exp \left(\frac{1}{k}\left(D^{\prime}+(\log \delta) I\right)\right)\right)^{k} \\
& =\left(M \exp \left(\frac{1}{k} \log \delta\right) \exp \left(\frac{1}{k} D^{\prime}\right)\right)^{k}=\delta\left(M D_{k}\right)^{k}
\end{aligned}
$$

and, on the other hand, calling $\gamma_{\delta D}$ to constant $\gamma$ for matrix $\delta D$, we have

$$
\gamma_{\delta D}=\exp \left(\mathbf{u}\left(D^{\prime}+(\log \delta) I\right) \mathbf{v}\right)=\exp \left(\mathbf{u} D^{\prime} \mathbf{v}+\log \delta\right)=\delta \gamma .
$$

If we take $\delta=\exp \left(-\left|\left\|D^{\prime} \mid\right\|\right)\right.$ we find, using (A5), that for every $k$,

$$
\left\|( \delta D ) _ { k } \left|\left\|=\left|\left\|\delta ^ { \frac { 1 } { k } } D _ { k } \left|\left\|=\delta^{\frac{1}{k}}\left|\left\|\exp \left(\frac{1}{k} D^{\prime}\right) \mid\right\| \leq \exp \left(-\frac{1}{k}\left|\left\|D^{\prime} \mid\right\|\right) \exp \left(\left|\left\|\frac{1}{k} D^{\prime} \mid\right\|\right)=1 .\right.\right.\right.\right.\right.\right.\right.\right.\right.\right.
$$

So, we can assume, without loss of generality, that matrix $D$ verifies \|\|$D_{k} \| \mid \leq 1$ and together with (A2) we obtain that

$$
\left\|\left(M D_{k}\right)^{i} \mid\right\| \leq 1 \quad \text { for every } i, k \in \mathbb{N} .
$$


Now, we decompose $\left(M D_{k}\right)^{i}$ using projector $\bar{M}$,

$$
\left(M D_{k}\right)^{i}=\bar{M}\left(M D_{k}\right)^{i}+(I-\bar{M})\left(M D_{k}\right)^{i} .
$$

First, we prove that $\left\|(I-\bar{M})\left(M D_{k}\right)^{k} \mid\right\|=O(1 / k)$,

$$
(I-\bar{M})\left(M D_{k}\right)^{i+1}=(I-\bar{M}) M D_{k}\left(M D_{k}\right)^{i}=(I-\bar{M}) M D_{k}\left(\bar{M}\left(M D_{k}\right)^{i}+(I-\bar{M})\left(M D_{k}\right)^{i}\right)
$$

using equalities (A1) and (A3), we get

$$
(I-\bar{M})\left(M D_{k}\right)^{i+1}=(M-\bar{M}) P_{k} \bar{M}\left(M D_{k}\right)^{i}+(M-\bar{M}) D_{k}(I-\bar{M})\left(M D_{k}\right)^{i}
$$

Denoting $w_{k}^{i}=\left|\left\|(I-\bar{M})\left(M D_{k}\right)^{i} \mid\right\|\right.$ and using (A2) and (A7), we obtain the following inequality:

$$
w_{k}^{i+1} \leq r||\left|P_{k}\right||||| \bar{M}|| \mid+r w_{k}^{i},
$$

which yields

$$
w_{k}^{i} \leq r^{i} w_{k}^{0}+||\left|P_{k}\right||||||\bar{M}||| \sum_{j=1}^{i} r^{j} \leq r^{i} w_{k}^{0}+||\left|P_{k}\right||| \frac{|\|\bar{M} \mid\| r}{1-r} .
$$

In particular,

$$
w_{k}^{k} \leq r^{k} w_{k}^{0}+||\left|P_{k}\right||| \frac{|\|\bar{M} \mid\| r}{1-r}=O\left(\frac{1}{k}\right)
$$

Second, we prove that $\left\|\left|\bar{M}\left(M D_{k}\right)^{k}-\left(1+(1 / k)\left(\mathbf{u} D^{\prime} \mathbf{v}\right)\right)^{k} \bar{M}\right|\right\|=O(1 / k)$, $\bar{M}\left(M D_{k}\right)^{i+1}=\bar{M} M D_{k}\left(M D_{k}\right)^{i}$ and using (A8) and (A4), we get

$$
\bar{M}\left(M D_{k}\right)^{i+1}=\bar{M}\left(I+(1 / k) D^{\prime}+N_{k}\right)\left(\bar{M}\left(M D_{k}\right)^{i}+(I-\bar{M})\left(M D_{k}\right)^{i}\right),
$$

and having in mind that $\bar{M} D^{\prime} \bar{M}=\left(\mathbf{u} D^{\prime} \mathbf{v}\right) \bar{M}$ and (A1), we deduce that

$$
\bar{M}\left(M D_{k}\right)^{i+1}=\left(1+\frac{1}{k}\left(\mathbf{u} D^{\prime} \mathbf{v}\right)\right) \bar{M}\left(M D_{k}\right)^{i}+\frac{1}{k} \bar{M} D^{\prime}(I-\bar{M})\left(M D_{k}\right)^{i}+\bar{M} N_{k}\left(M D_{k}\right)^{i},
$$

thus by induction, we obtain

$$
\begin{aligned}
\bar{M}\left(M D_{k}\right)^{k}= & \left(1+\frac{1}{k}\left(\mathbf{u} D^{\prime} \mathbf{v}\right)\right)^{k} \bar{M} \\
& +\sum_{j=0}^{k-1}\left(1+\frac{1}{k}\left(\mathbf{u} D^{\prime} \mathbf{v}\right)\right)^{j}\left(\frac{1}{k} \bar{M} D^{\prime}(I-\bar{M})\left(M D_{k}\right)^{k-1-j}+\bar{M} N_{k}\left(M D_{k}\right)^{k-1-j}\right) .
\end{aligned}
$$

So, we need to prove that

$$
\left\|\sum_{j=0}^{k-1}\left(1+\frac{1}{k}\left(\mathbf{u} D^{\prime} \mathbf{v}\right)\right)^{j}\left(\frac{1}{k} \bar{M} D^{\prime}(I-\bar{M})\left(M D_{k}\right)^{k-1-j}+\bar{M} N_{k}\left(M D_{k}\right)^{k-1-j}\right)\right\|=O\left(\frac{1}{k}\right) .
$$


From (A10), we get

$$
\sum_{i=0}^{k} w_{k}^{i} \leq \sum_{i=0}^{k} r^{i} w_{k}^{0}+\sum_{i=0}^{k}\left|\left\|P_{k}||\left|\frac{|\|\bar{M} \mid\| r}{1-r} \leq \frac{w_{k}^{0}}{1-r}+\frac{|\|\bar{M} \mid\| r}{1-r} k\right|\right\| P_{k}\right| \| \leq B<\infty
$$

and, using (A4) and (A7), we finally have

$$
\begin{aligned}
& \left\|\mid \sum_{j=0}^{k-1}\left(1+\frac{1}{k}\left(\mathbf{u} D^{\prime} \mathbf{v}\right)\right)^{j}\left(\frac{1}{k} \bar{M} D^{\prime}(I-\bar{M})\left(M D_{k}\right)^{k-1-j}+\bar{M} N_{k}\left(M D_{k}\right)^{k-1-j}\right)\right\| \\
& \leq \exp \left(1+\left|\mathbf{u} D^{\prime} \mathbf{v}\right|\right)\left(\frac{1}{k}||\left|\bar{M} D^{\prime}\right|\left|\sum_{j=0}^{k-1} w_{k}^{k-1-j}+\right||| \bar{M}\left|\left\|||\left|N_{k}\right|\left|\sum_{j=0}^{k-1}\right|\right\|\left(M D_{k}\right)^{k-1-j}\right| \|\right) \\
& \leq \exp \left(1+\left|\mathbf{u} D^{\prime} \mathbf{v}\right|\right)\left(\frac{1}{k}|| \bar{M} D^{\prime}|||B+||\bar{M}| \|||\left|N_{k}\right||| k\right)=O\left(\frac{1}{k}\right) .
\end{aligned}
$$

To finish the proof

$$
\begin{aligned}
& \|\|\left(M D_{k}\right)^{k}-\gamma \bar{M}\left|\left\|=\left|\left\|\bar{M}\left(M D_{k}\right)^{k}+(I-\bar{M})\left(M D_{k}\right)^{k}-\gamma \bar{M} \mid\right\|\right.\right.\right. \\
& \leq\left|\left\|(I-\bar{M})\left(M D_{k}\right)^{k}|\|+\|| \bar{M}\left(M D_{k}\right)^{k}-\left(1+\frac{1}{k}\left(\mathbf{u} D^{\prime} \mathbf{v}\right)\right)^{k} \bar{M}|| \mid\right.\right. \\
& \quad+\left|\left(1+\frac{1}{k}\left(\mathbf{u} D^{\prime} \mathbf{v}\right)\right)^{k}-\exp \left(\mathbf{u} D^{\prime} \mathbf{v}\right)\right|\left|\|\bar{M} \mid\|=O\left(\frac{1}{k}\right) .\right.
\end{aligned}
$$

